\title{
Post-Acute Sequelae of COVID-19
}

National Cancer Institute

\section{Source}

National Cancer Institute. Post-Acute Sequelae of COVID-19. NCI Thesaurus. Code C179263.

A constellation of signs and symptoms that can persist for weeks or months after acute infection with SARS-CoV-2, or appear weeks after infection. This condition may be characterized by fatigue, shortness of breath, difficulty focusing or concentrating ("brain fog"), sleep disorders, fevers, gastrointestinal symptoms, anxiety, and depression. 